\title{
An Activity-Subspace Approach for Estimating the Integrated Input Function and Relative Distribution Volume in PET Parametric Imaging
}

\author{
Peng Qiu, Member, IEEE, Z. Jane Wang, Member, IEEE, K. J. Ray Liu, Fellow, IEEE, and Zsolt Szabo
}

\begin{abstract}
Dynamic positron emission tomography (PET) imaging technique enables the measurement of neuroreceptor distributions corresponding to anatomic structures, and thus, allows imagewide quantification of physiological and biochemical parameters. Accurate quantification of the concentration of neuroreceptor has been the objective of many research efforts. Compartment modeling is the most widely used approach for receptor binding studies. However, current compartment-model-based methods often either require intrusive collection of accurate arterial blood measurements as the input function, or assume the existence of a reference region. To obviate the need for the input function or a reference region, in this paper, we propose to estimate the input function. We propose a novel concept of activity subspace, and estimate the input function by the analysis of the intersection of the activity subspaces. Then, the input function and the distribution volume (DV) parameter are refined and estimated iteratively. Thus, the underlying parametric image of the total DV is obtained. The proposed method is compared with a blind estimation method, iterative quadratic maximum-likelihood (IQML) via simulation, and the proposed method outperforms IQML. The proposed method is also evaluated in a brain PET dataset.
\end{abstract}

Index Terms-Activity subspace, positron emission tomography (PET).

\section{INTRODUCTION}

$\mathbf{T}$ HE FUNDAMENTAL aim of functional imaging such as positron emission tomography (PET) and single-photon emission computed tomography (SPECT) is to extract quantitative information about physiological and biochemical functions (e.g., physiological parameters) from medical images. PET is a nuclear imaging technique relying on the unique physics of radionuclides that decay via positron emission. PET in dynamic mode can produce sequential images of in vivo distribution of a radioligand over time. PET imaging has found many clinical applications, with substantial contributions to neurologic illnesses, oncology, and cardiovascular disease [1]. For example, using PET and a specific radioligand, the serotonin transporter (SERT) in the brain can be quantified to assess the integrity of serotonergic neurotransmission [2]. Of particular interest in this

Manuscript received October 29, 2007; revised April 15, 2008, June 2, 2008, and July 14, 2008. First published August 15, 2008; current version published January 4, 2009.

P. Qiu is with the Department of Radiology, Stanford University, Stanford, CA 94305 USA.

Z. J. Wang is with the Department of Electrical and Computer Engineering, University of British Columbia, BC V6T 1Z4, Canada (e-mail: zjanew@ece.ubc.ca).

K. J. R. Liu is with the Department of Electrical and Computer Engineering, University of Maryland, MD 20742 USA (e-mail: kjrliu@eng.umd.edu).

Z. Szabo is with the Department of Radiology, Johns Hopkins University Medical Institutions, Baltimore, MD 21287 USA (e-mail: zszabo@jhmi.edu).

Digital Object Identifier 10.1109/TITB.2008.2004485 paper is the image-wide quantification of the concentration of neuroreceptor.

For the purpose of neuroreceptor quantification, the PET time-activity data are commonly analyzed by fitting to a mathematical model. Compartmental model-based approaches are the most widely used for tracer kinetic modeling in dynamic imaging [3], [4]. The compartmental modeling approaches can be mainly classified into two categories, namely, invasive and noninvasive, on the basis of whether arterial blood sampling is required. In the invasive approaches, a sequence of arterial plasma samples is used as the input function in the kinetic model [5], [6]. Though invasive models have some advantages, arterial plasma samples are often difficult to obtain or measure accurately, and such invasive measurement represents a limited, but not negligible risk of complications including thrombosis, infection, and nerve injury [7]. Therefore, there has been increasing interest in noninvasive techniques.

Noninvasive techniques in the literature can be further classified depending on whether a reference region is needed. Examples of reference-region-based methods include [8] where the time-activity curve (TAC) from carotid artery regions of interest (ROI) is used as the input function, and [9], [10] where a two-compartment model is assumed for the reference region. The reference region models obviate the need for input function by obtaining the parameters as a function of the reference region TAC. Another noninvasive research direction is to estimate both the kinetic parameters and the input function simultaneously. For example, in [11] and [12], the input function is assumed to follow a mathematical formula, with several parameters to be determined. The parameters for the input function and the kinetic parameters are estimated simultaneously through weighted nonlinear least-square (LS) method. Three blind identification schemes in [13] are examined and compared, namely, the cross-relations (CRs) method, the iterative quadratic maximum likelihood method (IQML), and the eigenvector-based algorithm (EVAM). These methods estimate kinetic parameters without requiring the knowledge of the input function. The input function can be obtained based on the observed TACs and the estimated kinetic parameters.

In this study, we revisit a novel noninvasive approach first proposed by our group [14], called the activity-subspace approach, and also, referred to as intersectional searching algorithm (ISA) in [15]. The proposed activity-subspace approach does not assume any reference regions, and thus, falls into the category that estimates both the kinetic parameters and the input function. Compared with [11] and [12], the proposed activitysubspace approach does not assume any mathematical structure 


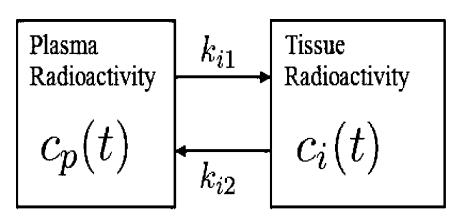

(a)

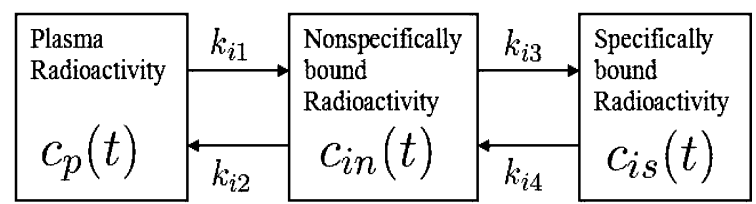

(b)

Fig. 1. Two-compartment model and three compartment model for voxel $i$ in the region of interest.

of the input function. Different from the methods discussed in [13], the proposed approach first estimates the input function through the intersection of activity subspaces. Then, with the estimated input function, the distribution volume (DV) parameters are iteratively estimated, and the estimated input function is refined at the same time. The basic idea is intuitively explained as follows. Since the TAC of each voxel is determined by the plasma input function and the dynamics of this voxel, it can be derived from the compartmental models that for each voxel, a 2-D activity subspace can be defined from its TAC; the integral of the input function belongs to the activity subspace. It is noted that a common input function is shared by all the voxels. Therefore, theoretically, given several different voxels and their corresponding activity subspaces, the integral of the common input function belongs to all these different activity subspaces, and thus, can be estimated by finding the intersection of the activity subspaces. Very recently, Naganawa et al. [15] proposed a method, called robust EPISA, for robust extraction of the cumulative integral of the input by jointly using ISA and clustering, and found successful applications in real PET analysis. It was shown that the calculated neuroreceptor images by the robust EPISA have a quality equivalent to that using the measured input after metabolite correction [15]. However, despite the promise of EPISA, there are still two disadvantages remained with the current activity-subspace-based approaches: one is that it is shown to be sensitive to noise; the other is that the monotonicity of the estimated integral is not guaranteed. To overcome these two problems, in this paper, we propose to use a novel clustering algorithm [16] jointly with the activity-subspace approach, which can efficiently reduce the noise in PET data. We also propose an iterative LS procedure that refines the estimated integral obtained by the activity-subspace approach, where the monotonicity of the estimated integral is enforced in the iterative procedure.

This paper is organized as follows. In Section II, we first describe the models in the voxel domain, including the twocompartment model, three-compartment model, and the graphical analysis (GA) plot of [17]; we then formulate the problem. In Section III, we present the proposed activity-subspace approach to estimate the input function and the parametric image of the total DV. In Section IV, data simulations are put into operation. The performance of the proposed scheme is examined using brain PET datasets in Section V, followed by Discussions and Conclusions.

\section{SYSTEM MODEL AND FORMULATION}

Accurate estimation of parametric images in neuroreceptor studies often requires fitting the TACs to a mathematical model. Compartment models are the most popular models used for physiologically based quantification of the neuroreceptor concentration. In the literature, the in vivo tracer kinetics are often represented by a serial compartmental model [3]. Measures such as binding potential (BP) and DV are then often calculated based on the model parameters. A simple compartment model is the two-compartment model, as illustrated in Fig. 1(a). The $i$ th voxel within the organ of interest is modeled by two compartments. One compartment $c_{p}(t)$ represents the radiotracer concentration in arterial blood at time $t$, and the other represents radiotracer concentration in the tissue compartment $c_{i}(t)$. The two-compartment model can be mathematically written as a linear differential equation

$$
\frac{\mathrm{d} c_{i}(t)}{\mathrm{d} t}=k_{i 1} c_{p}(t)-k_{i 2} c_{i}(t)
$$

where the rate constants $k_{i 1}$ and $k_{i 2}$ represents blood-tissue exchange parameters for the $i$ th voxel. Equation (1) can be integrated and rearranged into the following form:

$$
\int_{0}^{T} c_{i}(t) d t=\frac{k_{i 1}}{k_{i 2}} \int_{0}^{T} c_{p}(t) d t-\frac{1}{k_{i 2}} c_{i}(T) .
$$

Another widely used compartment model is the threecompartment model. For instance, in serotonin transporter SERT imaging, brain regions containing receptors have the minimal number of three compartments. The three-compartment model is illustrated in Fig. 1(b). The $i$ th voxel within the organ of interest is modeled by three compartments, where $c_{p}(t)$ represents radiotracer concentration in arterial blood, $c_{i n}(t)$ is the radioactivity in the nonspecific binding compartment, and $c_{i s}(t)$ is the radioactivity in specific binding compartment. The arrows represent the direction by which radioligand can move between compartments with model parameters $k_{i 1}, k_{i 2}, k_{i 3}, k_{i 4}$. Mathematically, Fig. 1(b) can be represented by two linear differential equations:

$$
\begin{aligned}
& \frac{\mathrm{d} c_{i s}(t)}{\mathrm{d} t}=k_{i 3} c_{i n}(t)-k_{i 4} c_{i s}(t) \\
& \frac{\mathrm{d} c_{i n}(t)}{\mathrm{d} t}=k_{i 1} c_{p}(t)-\left(k_{i 2}+k_{i 3}\right) c_{i n}(t)+k_{i 4} c_{i s}(t) .
\end{aligned}
$$

In PET imaging experiments, $c_{i s}(t)$ and $c_{i n}(t)$ cannot be measured separately. The observed receptor activity in the $i$ th brain voxel is the total concentration $c_{i}(t)=c_{i s}(t)+c_{i n}(t)$. Following the same derivation as in [18], the dynamics of each voxel $i$ at time $T$ satisfies the following equation:

$$
\begin{aligned}
c_{i}(T)= & \gamma_{i 1} \int_{0}^{T} \int_{0}^{s} c_{p}(t) d t d s+\gamma_{i 2} \int_{0}^{T} \int_{0}^{s} c_{i}(t) d t d s \\
& +\gamma_{i 3} \int_{0}^{T} c_{i}(t) d t+\gamma_{i 4} \int_{0}^{T} c_{p}(t) d t
\end{aligned}
$$


where the parameters $\gamma_{i 1} \sim \gamma_{i 4}$ are uniquely determined by the parameters $k_{i 1} \sim k_{i 4}$.

In the GA plot introduced by Logan et al. [17], an asymptotically linear relationship can be observed for the threecompartment model. For voxel $i$

$$
\frac{\int_{0}^{T} c_{i}(t) d t}{c_{i}(T)}=V_{i T} \frac{\int_{0}^{T} c_{p}(t) d t}{c_{i}(T)}+b_{i}
$$

where the slope $V_{i T}$ is the total DV (which is expressed as $\left.\left(k_{i 1} / k_{i 2}\right)\left(1+k_{i 3} / k_{i 4}\right)\right)$, and $b_{i}$ is the intercept that becomes constant for $T>t^{*}$. This linear relationship is asymptotically valid for the late part of the dynamic PET experiment. By rearranging (4), for $T>t^{*}$, we can obtain a form that is very similar to $(2)$

$$
\int_{0}^{T} c_{i}(t) d t=V_{i T} \int_{0}^{T} c_{p}(t) d t+b_{i} c_{i}(T) .
$$

Therefore, this relationship (5) is valid both for the twocompartment model and three-compartment model when $T>t^{*}$.

In existing literature, assuming that the input function $c_{p}(t)$ is known, models in (2), (3), and (5) allow estimation of kinetic parameters by multilinear regression analysis of the voxel TACs [18]. However, in the problem of interest here, the knowledge of the input function $c_{p}(t)$ is not available. Therefore, our goal is to estimate both the input function $c_{p}(t)$ and the kinetic parameters.

We now describe the basic idea by first introducing the discrete version of the model in (5). In practice, the PET images are generally acquired with increasing time intervals. Let $\mathbf{t}=$ $\left\{t_{l}, t_{l+1}, \ldots, t_{n}\right\}$ denotes the sampling time points that are later than $t^{*}$. By evaluating the terms in (5) at time points in $\mathbf{t}$, we define the following vectors, $\mathbf{c}_{i}=\left[c_{i}\left(t_{l}\right), c_{i}\left(t_{l+1}\right), \ldots, c_{i}\left(t_{n}\right)\right]^{T}$, $\mathbf{c}_{i}^{\text {int }}=\left[\int_{0}^{t_{l}} c_{i}(t) d t, \int_{0}^{t_{l+1}} c_{i}(t) d t, \ldots, \int_{0}^{t_{n}} c_{i}(t) d t\right]^{T}$, and $\mathbf{c}_{p}^{\text {int }}=$ $\left[\int_{0}^{t_{l}} c_{p}(t) d t, \int_{0}^{t_{l+1}} c_{p}(t) d t, \ldots, \int_{0}^{t_{n}} c_{p}(t) d t\right]^{T}$. Equation (5) can be written in a vector form

$$
\mathbf{c}_{i}^{\text {int }}=V_{i T} \mathbf{c}_{p}^{\text {int }}+b_{i} \mathbf{c}_{i}
$$

where the superscript int represents the single integral operation. Therefore, in the voxel domain, we can observe the following relationship for each voxel:

$$
\mathbf{c}_{p}^{\mathrm{int}}=\frac{-b_{i}}{V_{i T}} \mathbf{c}_{i}+\frac{1}{V_{i T}} \mathbf{c}_{i}^{\mathrm{int}} .
$$

From (7), it can be observed that the integral of the input function $\mathbf{c}_{p}^{\text {int }}$ can be written as the linear combination of the $i$ th voxel's TAC and the integral of the $i$ th voxel's TAC. For the $i$ th voxel, we define the space spanned by $\mathbf{c}_{i}$ and $\mathbf{c}_{i}^{\text {int }}$ as its activity subspace, which is of dimension two. From (7), the integral of the input function $\mathbf{c}_{p}^{\text {int }}$ lies in the activity subspace of the $i$ th voxel. Since a common input function is shared by all the voxels, the integral of the input function belongs to the activity subspaces of all voxels within the organ of interest. Therefore, we can estimate $\mathbf{c}_{p}^{\text {int }}$ by finding the intersection of the different activity subspaces. After obtaining the estimate of $\mathbf{c}_{p}^{\text {int }}$, we can apply iterative LS method to iteratively estimate the kinetic parameters in (7) and refine the estimate of $\mathbf{c}_{p}^{\mathrm{int}}$.

\section{Proposed Scheme}

In this section, the proposed activity-subspace approach is described in detail. In order to estimate the integral of the input function based on the noisy PET measurements, the problem of finding the intersection of different activity subspaces is formulated as an optimization problem. To reduce the noise effect, mixture principal component analysis (mPCA) [19] is employed to group the voxel TACs into several clusters. Since each cluster contains voxels with similar TACs and kinetic parameters, the cluster average TAC will follow the model in (7). Through averaging, the noise effect is reduced. The activity-subspace approach will operate on the cluster average TACs, estimating the integral of the input function by finding the intersection between the activity subspaces defined by cluster average TACs.

\section{A. Activity-Subspace Intersection for Estimating the Integral of the Input Function}

In (7), assuming that $\mathbf{c}_{i}$ and $\mathbf{c}_{i}^{\text {int }}$ are noise free, we can see that the vector $\mathbf{c}_{p}^{\text {int }}$ lies in the subspace spanned by $\mathbf{c}_{i}$ and $\mathbf{c}_{i}^{\text {int }}$, which is defined as voxel $i$ 's activity subspace. For any two different voxels $i$ and $j$ with different kinetic parameters, ideally the vector $\mathbf{c}_{p}^{\text {int }}$ should belong to both the activity subspace spanned by $\mathbf{c}_{i}$ and $\mathbf{c}_{i}^{\text {int }}$ and the activity subspace spanned by $\mathbf{c}_{j}$ and $\mathbf{c}_{j}^{\text {int }}$. Equivalently, in the ideal noise-free situation, the intersection of the two activity subspaces is a 1-D subspace (a line), which defines the direction of the vector $\mathbf{c}_{p}^{\text {int }}$. This observation motivates us to estimate the vector $\mathbf{c}_{p}^{\text {int }}$ by exploring the intersection of the activity subspaces.

Another observation is that although the direction of the vector $\mathbf{c}_{p}^{\text {int }}$ can be determined through the intersection of activity subspaces, we are not able to determine the length of the vector. In other words, we are able to estimate the shape of the curve $\int_{0}^{T} c_{p}(t) d t$, but the amplitude remains unknown. As far as the DV parametric image is concerned, not knowing the amplitude will not cause a problem. For example, if we determine the direction of the vector $\mathbf{c}_{p}^{\text {int }}$ from the intersection of activity subspaces and constraint the length of the vector to be 1 , the estimated integral of input function $\hat{\mathbf{c}}_{p}^{\text {int }}$ will be a scaled version of the integral of the true input function, i.e., $\hat{\mathbf{c}}_{p}^{\mathrm{int}}=\eta \mathbf{c}_{p}^{\mathrm{int}}$, where $\eta$ is a scalar. If we use $\hat{\mathbf{c}}_{p}^{\text {int }}$ to estimate the kinetic parameters in (6) using linear regression, the parameter $b_{i}$ will be estimated accurately, and the estimated total DV $\hat{V}_{i T}$ will be scaled by a factor of $1 / \eta$, i.e., $\hat{V}_{i T}=1 / \eta V_{i T}$. Since the estimated total DV is scaled by the same factor for all voxels, the total DV parametric image will not be affected. Therefore, in the proposed method, we constrain the estimated integral of the input function to be a vector of unit length, i.e., $\left|\hat{\mathbf{c}}_{p}^{\text {int }}\right|=1$.

In practice, $c_{i}(t)$ 's are noisy measurements, and thus, the integral of $c_{i}(t)$ appears as a noise source too. The noisy nature of $c_{i}(t)$ certainly will affect the precision of the activity subspace spanned by $\mathbf{c}_{i}$ and its integral $\mathbf{c}_{i}^{\text {int }}$, and thus, the intersection of the activity subspaces. As mentioned earlier, in the ideal noise-free case, the intersection line between $\operatorname{span}\left(\mathbf{c}_{i}, \mathbf{c}_{i}^{\text {int }}\right)$ and $\operatorname{span}\left(\mathbf{c}_{j}, \mathbf{c}_{j}^{\text {int }}\right)$ defines the integral of the input function $\mathbf{c}_{p}^{\text {int }}$. However, because of the measurement noise, both activity 
subspaces are disturbed and the intersection line may no longer exist. Therefore, we propose to approximate the intersection by the following. In the ideal noise-free case, the intersection between $\operatorname{span}\left(\mathbf{c}_{i}, \mathbf{c}_{i}^{\text {int }}\right)$ and $\operatorname{span}\left(\mathbf{c}_{j}, \mathbf{c}_{j}^{\text {int }}\right)$ can be equivalently regarded as a pair of lines, one in each activity subspace, that share maximum correlation. Through a similar methodology, with the presence of measurement noise, we can find a pair of lines, such that one belongs to $\operatorname{span}\left(\mathbf{c}_{i}, \mathbf{c}_{i}^{\text {int }}\right)$, the other belongs to $\operatorname{span}\left(\mathbf{c}_{j}, \mathbf{c}_{j}^{\text {int }}\right)$, and this pair of lines share maximum correlation. The average of this pair of lines could be a reasonable approximation of the intersection line in the ideal case, which is the integral of the input function.

Mathematically, assume that vectors $\mathbf{v}_{i 1}$ and $\mathbf{v}_{i 2}$ are the orthonormal basis of the subspace $\operatorname{span}\left(\mathbf{c}_{i}, \mathbf{c}_{i}^{\text {int }}\right)$. Then, any unit length vector $\mathbf{u}_{i}$ in $\operatorname{span}\left(\mathbf{c}_{i}, \mathbf{c}_{i}^{\text {int }}\right)$ can be written as

$$
\mathbf{u}_{i}=\cos \left(\theta_{i}\right) \mathbf{v}_{i 1}+\sin \left(\theta_{i}\right) \mathbf{v}_{i 2}
$$

where $\theta_{i} \in[0,2 \pi)$. Therefore, the problem of finding the approximation of the intersection line between two activity subspaces can be formulated as the following optimization problem:

$$
\begin{aligned}
& \max _{\theta_{i}, \theta_{j}} \mathbf{u}_{i}^{T} \mathbf{u}_{j} \\
& \text { s.t. }\left\{\begin{array}{l}
\mathbf{u}_{i}=\cos \left(\theta_{i}\right) \mathbf{v}_{i 1}+\sin \left(\theta_{i}\right) \mathbf{v}_{i 2} \\
\mathbf{u}_{j}=\cos \left(\theta_{j}\right) \mathbf{v}_{j 1}+\sin \left(\theta_{j}\right) \mathbf{v}_{j 2} \\
\theta_{i} \in[0,2 \pi) \quad \theta_{j} \in[0,2 \pi)
\end{array}\right.
\end{aligned}
$$

where $\mathbf{v}_{i 1}$ and $\mathbf{v}_{i 2}$ are the orthonormal basis of the activity subspace $\operatorname{span}\left(\mathbf{c}_{i}, \mathbf{c}_{i}^{\text {int }}\right)$, and $\mathbf{v}_{j 1}$ and $\mathbf{v}_{j 2}$ are the orthonormal basis of the activity subspace $\operatorname{span}\left(\mathbf{c}_{j}, \mathbf{c}_{j}^{\text {int }}\right)$. This maximization problem can be solved by grid search of possible values of $\theta_{i}$ and $\theta_{j}$ over the range of $[0,2 \pi)$ (in the current implementation, the grid search interval is 0.001 , meaning that we examine $\theta_{i}$ and $\theta_{j}$ that take any values, which are multiples of 0.001 ). Assume that $\theta_{i}=\theta_{i}^{*}$ and $\theta_{j}=\theta_{j}^{*}$ maximizes the objective, the corresponding $\mathbf{u}_{i}\left(\theta_{i}^{*}\right)$ and $\mathbf{u}_{j}\left(\theta_{j}^{*}\right)$ are the pair of lines, from activity subspaces of voxel $i$ and $j$, that maximize the correlation. Note that if $\mathbf{u}_{i}\left(\theta_{i}^{*}\right)$ and $\mathbf{u}_{j}\left(\theta_{j}^{*}\right)$ is the solution to the maximization problem in (9), so does $-\mathbf{u}_{i}\left(\theta_{i}^{*}\right)$ and $-\mathbf{u}_{j}\left(\theta_{j}^{*}\right)$. We choose one of the two solutions based on the fact that the elements of $\mathbf{c}_{p}^{\text {int }}$ are all positive. If the number of positive elements is greater than the number of negative elements in vectors $\mathbf{u}_{i}\left(\theta_{i}^{*}\right)$ and $\mathbf{u}_{j}\left(\theta_{j}^{*}\right)$, the integral of the input function is approximated by $\hat{\mathbf{c}}_{p}^{\mathrm{int}}=1 / 2\left(\mathbf{u}_{i}\left(\theta_{i}^{*}\right)+\mathbf{u}_{j}\left(\theta_{j}^{*}\right)\right)$. Otherwise, the integral of the input function is approximated by $\hat{\mathbf{c}}_{p}^{\text {int }}=1 / 2\left(-\mathbf{u}_{i}\left(\theta_{i}^{*}\right)-\mathbf{u}_{j}\left(\theta_{j}^{*}\right)\right)$.

\section{B. Clustering of Voxels}

To yield a fine approximation of the integral of the input function, it is desirable to reduce the noise level in the voxel TACs, which are used to define the activity subspaces. To achieve this purpose, we cluster the voxels into several clusters according to their TACs. This idea is similar with working on the virtual regional TACs (e.g., each cluster represents a virtual regional TAC, which is not necessarily a spatially contiguous brain region). After clustering, each cluster is represented by the average TAC based on which the cluster's activity subspace is defined.
The integral of the input function can be estimated from the intersection of the activity subspaces of different clusters.

Clustering voxels can be regarded as identifying different voxel activity patterns where each activity pattern can be represented by a different underlying model. As demonstrated in many areas, the idea of mPCA has been a promising framework to deal with such problems by modeling the underlying nonlinearity and complexity with a mixture of local linear submodels [16]. In our study, we propose to apply the mPCA approach for clustering.

The concepts in mPCA is related with the PET parametric imaging problem as follows. In the mPCA approach, a set of probabilistic PCA models is introduced by associating a probability density with the conventional PCA model. Assume that there exist $K$ clusters of functionally different voxels, whose activity patterns are different. mPCA describes each cluster by a probabilistic PCA model. The relative size of each cluster is modeled by a set of prior probabilities. Therefore, a voxel $i$ with probability $p(k)$ (prior) belongs to the $k$ th cluster, and thus, can be represented by the $k$ th probabilistic PCA model

$$
y_{i}=W_{k} x+\mu_{k}+\epsilon_{k}
$$

where $y_{i}$ is voxel $i$ 's TAC. $x$ is assumed to be an independent Gaussian vector with unit variance, $x \sim N(0, I)$ and dimension $q$. The columns of the matrix $W_{k}$ are the $q$ principal components of the activity pattern in the $k$ th cluster. The principal components are not necessarily unitary, so that the energy of each component can be taken care of in the $W_{k}$ matrix. The parameter $\mu_{k}$ represents the mean activity pattern, and $\epsilon_{k}$ represents measurement noise. For the case of isotropic noise $\epsilon_{k} \sim N\left(0, \sigma_{i}^{2} I\right)$, the conditional probability of observing TAC, $y_{i}$ can be written as follows:

$$
p\left(y_{i} \mid x, k\right)=\left(2 \pi \sigma_{k}^{2}\right)^{-d / 2} e^{-\left(1 / 2 \sigma_{k}^{2}\right)\left\|y_{i}-W_{k} x-\mu_{k}\right\|^{2}} .
$$

Integrating the previous equation over the distribution of $x$, we obtain the distribution of observation $y_{i}$ given the $k$ th probabilistic PCA model

$$
\begin{aligned}
p\left(y_{i} \mid k\right) & =\int p\left(y_{i} \mid x, k\right) p(x \mid k) d x \\
& =(2 \pi)^{-d / 2}\left|C_{k}\right|^{-1 / 2} e^{-(1 / 2)\left(y_{i}-\mu_{k}\right)^{T} C_{k}^{-1}\left(y_{i}-\mu_{k}\right)}
\end{aligned}
$$

where $C_{k}=\sigma_{k}^{2} I+W_{k} W_{k}^{T}$. Given the prior probabilities $p(k)$ of the set of $K$ probabilistic PCA models, according to Bayes rule, the posterior probability of the $k$ th model can be expressed as

$$
p\left(k \mid y_{i}\right)=\frac{p\left(y_{i} \mid k\right) p(k)}{\sum_{k=1}^{K} p\left(y_{i} \mid k\right) p(k)} .
$$

The posterior probability can be used for the purpose of clustering.

As in [19], an EM algorithm of mPCA is derived. In the E-step, given the observed TACs and model parameters from previous iteration, the posterior probability of the $k$ th model can be calculated. In the M-step, the model parameters are updated based on the previous model parameters and the posterior probabilities. The detailed EM algorithm is described in the Appendix. When the algorithm converges, for each voxel, we obtain 
the posterior probability of the voxel belonging to each probabilistic PCA model conditioning on the observed TAC. Based on the posterior probabilities, voxels are grouped into clusters. For each cluster, the average TAC is calculated, based on which the activity subspace of the cluster is defined. The integral of the input function is estimated from the intersection of activity subspaces of different clusters, using the method described in Section III-A.

\section{Iterative LS for Refining the Estimated Integral}

After voxel clustering, the integral of the input function is estimated from the intersection of activity subspaces defined by the clusters' average TACs. We extend the analysis to the voxel domain to further improve the accuracy of the estimated integral. Since each voxel TAC is a function of the same input function, we explore this property using the iterative LS method. For each voxel $i$, the noisy model of (6) can be expressed as

$$
\mathbf{c}_{i}=\frac{-V_{i T}}{b_{i}} \mathbf{c}_{p}^{\mathrm{int}}+\frac{1}{b_{i}} \mathbf{c}_{i}^{\mathrm{int}}+\mathbf{n}_{i}=\left(\mathbf{c}_{p}^{\mathrm{int}}, \mathbf{c}_{i}^{\mathrm{int}}\right)\left(\begin{array}{c}
\frac{-V_{i T}}{b_{i}} \\
\frac{1}{b_{i}}
\end{array}\right)+\mathbf{n}_{i}
$$

where the noise term $\mathbf{n}_{i}$ contains the measurement noise, the error from numerical integration, and the model mismatch error due to the asymptotically linear assumption. Define the observation matrix $\mathbf{C}=\left[\mathbf{c}_{1}, \ldots, \mathbf{c}_{N}\right], \mathbf{S}=\left[\mathbf{c}_{p}^{\text {int }}, \mathbf{c}_{1}^{\text {int }}, \ldots, \mathbf{c}_{N}^{\text {int }}\right]$, the coefficient matrix A with $A(1, i)=-V_{i T} / b_{i}, A(i+1, i)=1 / b_{i}$ and all other elements being zero, and the noise matrix $\mathbf{N}=$ $\left[\mathbf{n}_{1}, \ldots, \mathbf{n}_{N}\right]$. Combining (14) of multiple voxels, we obtain the block formulation

$$
\mathbf{C}=\mathbf{S A}+\mathbf{N}
$$

In order to estimate the kinetic parameters $V_{i T}, b_{i}$, the LS formulation yields the following minimization problem:

$$
\min _{\mathbf{c}_{p}^{\text {int }},\left\{V_{i T}, b_{i}\right\}}\|\mathbf{C}-\mathbf{S A}\|_{F}^{2} .
$$

Searching for the global minimizer is computationally prohibitive even for modest number of voxels under consideration. To achieve an affordable computational cost, we apply the iterative LS method. The basic idea is that we start from the initial estimate of $\mathbf{c}_{p}^{\text {int }}$ based on the intersection of activity subspaces of voxel clusters, as described in Sections III-A and III-B. Based on the estimated integral of input function, the coefficients in A can be estimated by LS regression. Using the estimate of $\mathbf{A}$, which is the estimated $\left\{V_{i T}, b_{i}\right\}$ for all voxels, the integral of the input function can be calculated from (7) for each voxel. The estimate of $\mathbf{c}_{p}^{\text {int }}$ can be updated based on the calculated integrals from all the voxels. Note that the initial estimate is obtained by the intersection of activity spaces, where the monotonicity is not guaranteed. In the iterative LS procedure, the monotonicity can be easily enforced by updating $\mathbf{c}_{p}^{\text {int }}$ based on the average of the calculated integrals that are monotonically increasing. The process iterates until convergence, when the difference between estimated $\mathbf{c}_{p}^{\text {int }}$ in consecutive iterations is below a certain threshold.

The minimization problem in (16) has many local minimizers. Therefore, in order to solve for the global minimizer, a good initialization is required. In our scheme, the activity-subspace approach is employed to obtain the initial estimate of $\mathbf{c}_{p}^{\text {int }}$, which aims at finding the estimate of $\mathbf{c}_{p}^{\text {int }}$ that fits the activity subspaces defined by several voxel clusters. We believe that such an initial estimate is naturally a good choice as it follows the underlying signal model. During the iterative LS procedure, we iteratively refine the estimate of the integral of the input function, the kinetic parameters are estimated at the same time. (Please note that, although we express the formulation in matrix form, the estimates are obtained in a voxel-by-voxel fashion.)

\section{Summary of the Proposed Scheme}

In summary, the following steps are taken.

1) Initialization: We apply the activity-subspace approach to obtain the initial estimate of the integral of the input function. More specifically

a) Preprocess time-activity data and identify voxels that belong to the brain region.

b) Cluster the voxels TACs into $M$ clusters using mPCA. A reasonable value of $M$ should be chosen. Based on our observations, $M=2$ could be a good choice. Denote the average cluster TACs as $\mathbf{x}_{j}, j=1, \ldots, M$, and their single integrals as $\mathbf{x}_{j}^{\text {int }}$.

c) For each pair $(i, j)$, where $i, j \in[1, M]$, the intersection of the activity subspaces $\operatorname{span}\left(\mathbf{x}_{i}, \mathbf{x}_{i}^{\text {int }}\right)$ and $\operatorname{span}\left(\mathbf{x}_{j}, \mathbf{x}_{j}^{\text {int }}\right)$ is obtained by (9). The intersections are the estimates of the integral of the input function. The average of them will serve as the initial estimate of $\mathbf{c}_{p}^{\text {int }}$.

2) Iterative Refinement: With the initial estimate of $\mathbf{c}_{p}^{\text {int }}$, the iterative LS is applied to further improve the estimation accuracy. At each iteration

a) Given the estimated $\mathbf{c}_{p}^{\text {int }}$ from the previous iteration, for each voxel $i$, the coefficients $V_{i T}$ and $b_{i}$ are estimated using LS regression.

b) For each voxel, given the coefficients $\left\{V_{i T}, b_{i}\right\}$, the integral of the input function can be calculated from (7).

c) The estimate of $\mathbf{c}_{p}^{\text {int }}$ is updated by the average of the calculated integrals that are monotonically increasing. The estimate of $\mathbf{c}_{p}^{\mathrm{int}}$ is then normalized to a unit length vector.

d) Iterate until convergence, the difference between estimated $\mathbf{c}_{p}^{\text {int }}$ in consecutive iterations is below a certain threshold.

Note that, if the data dynamic follows the two-compartment model, (6) holds during the entire experiment. In this case, $t^{*}=0$ and the integral of the input function during the entire experiment can be estimated. If the data follow the threecompartment model, (6) is an asymptotic relationship that holds for the later duration of the experiment. In this case, $t^{*}>0$, and for the integral of the input function, only the time points later than $t^{*}$ can be estimated. The value of $t^{*}$ needs to be carefully chosen. The choice of $t^{*}$ is a tradeoff between bias and variance. Since the GA plot is an asymptotical relationship for the later part of the PET experiment, if we choose a larger $t^{*},(6)$ and (7) 


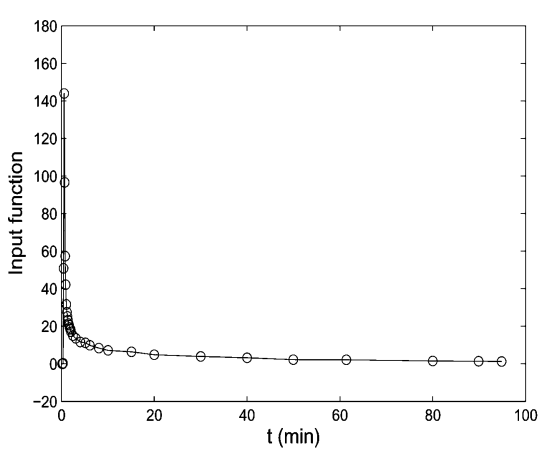

(a)

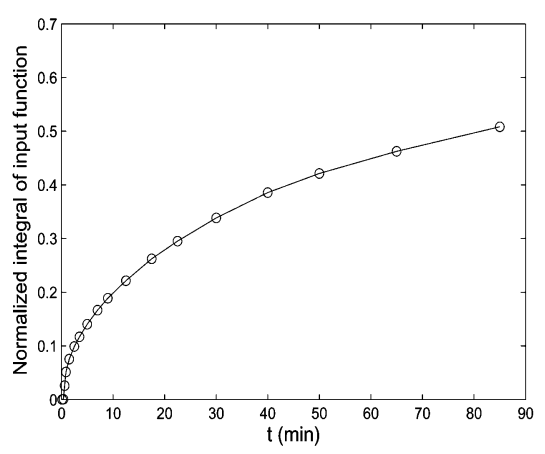

(c)

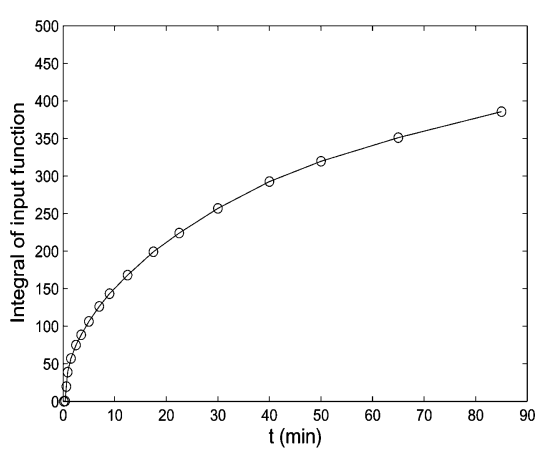

(b)

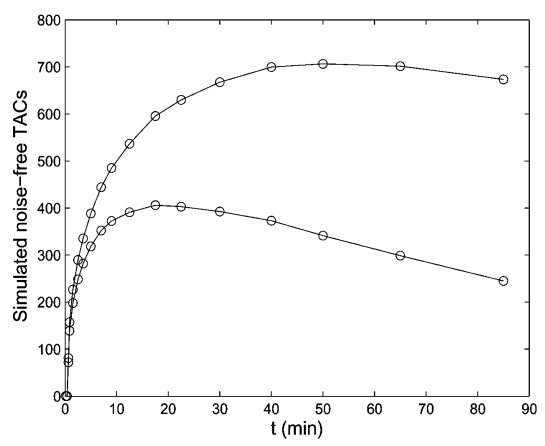

(d)

Fig. 2. (a) Input function used in the simulation. (b) Integral of the input function, resampled at the time points that correspond to the sampling point of voxel activity. (c) Normalized integral of the input function. (d) TACs of the two simulated voxels.

will better fit the TACs. On the other hand, since only the time points after $t^{*}$ are used to define the activity subspaces, larger $t^{*}$ will reduce the available time points, and thus, decrease the robustness of the estimated activity subspaces.

\section{Simulation Results}

In the previous section, a novel idea of estimating the input function from activity subspaces is proposed. In this section, we examine the performance of the activity-subspaces approach through simulation.

The time activities of two voxels are simulated by the three-compartment model. The kinetic parameters of the simulated voxels are $k_{11}=3.6247, k_{12}=0.0659, k_{13}=$ $0.0306, \quad k_{14}=0.0372$ and $k_{21}=4.0716, \quad k_{22}=0.0387$, $k_{23}=0.0507, k_{24}=0.0229$, respectively. The parameters are derived from a brain PET study of healthy control subjects using C-11 labeled DASB (3- ${ }^{11} \mathrm{C}$-amino-4-(2 dimethylaminomethylphenylsulfanyl) benzonitrile), as described in Section V. The input function used in the simulation is shown in Fig. 2(a), where the circles indicate the time points when the input function is sampled. Similar with the brain PET study in Section V, the activity of the simulated voxels are measured at 18 serial time points, which are different from the sampling time points of the input function. Since our vector form formulation in (7) evaluates the integral of the input function at the time points that correspond to the measured voxel activity, we integrate the input function and resample it at the 18 time points of the voxel activity measurement, as shown in
Fig. 2(b). Moreover, in Section III-A, it is mentioned that the activity-subspace approach is only able to estimate the direction of $\mathbf{c}_{p}^{\text {int }}$ (the shape of the integral of the input function), and the estimated integral of the input function $\hat{\mathbf{c}}_{p}^{\text {int }}$ is constrained to be a unit length vector. Therefore, a successful estimation $\hat{\mathbf{c}}_{p}^{\text {int }}$ will equal to the normalized integral of the input function, denoted as $\overline{\mathbf{c}}_{p}^{\text {int }}=\left(1 /\left|\mathbf{c}_{p}^{\text {int }}\right|\right) \mathbf{c}_{p}^{\text {int }}$. The normalized integral of the input function is shown in Fig. 2(c), which is what we plan to estimate using the activity-subspace approach. The value of parameter $t^{*}$ is chosen to be $t^{*}=20$. In the simulated data and the real PET data, there are six time points after $t^{*}=20$. We choose $t^{*}$ to be as large as possible, while guaranteeing enough time points for estimating the activity subspaces and the integral of the input function.

Using the input function in Fig. 2(a) and the kinetic parameters listed earlier, we simulate the TACs of the two voxels under the noise free case, shown in Fig. 2(d). Base on the noise-free TACs and the integral of the input function, the kinetic parameters in (6) can be obtained through linear regression. We also calculate the kinetic parameters based on the noise-free TACs and the normalized integral of the input function. The results are shown in Table I. In this table, we can see that the relative ratios between the two voxels' DVs in both cases are the same. In the case where normalized integral of the input function is used, the calculated distribution parameters of the two voxels are scaled by the same factor, compared with the case where the true integral of the input function is used. Therefore, for the purpose of estimating DV parametric image, the knowledge of the normalized integral of the input function will lead to the same 
TABLE I

Kinetic Parameters of Simulated Voxels, Calculated Based on Two Cases: Noise Free TaCs and True Integral of the InPut Function; NOISE-FREE TACS AND NORMALIZED INTEGRAL OF THE INPUT FUNCTION

\begin{tabular}{|c|c|c|c|}
\hline & voxel 1 & voxel 2 & ratio \\
\hline regression based on noise free TACs and & $V_{1 T}=95.0525$ & $V_{2 T}=294.9598$ & $V_{1 T} / V_{2 T}=0.3223$ \\
true integral of the input function & $b_{1}=-34.5938$ & $b_{2}=-90.2277$ & \\
\hline regression based on noise free TACs and & $V_{1 T}=72123.4$ & $V_{2 T}=223807.7$ & $V_{1 T} / V_{2 T}=0.3223$ \\
normalized integral of the input function & $b_{1}=-34.5938$ & $b_{2}=-90.2277$ & \\
\hline
\end{tabular}

performance with the case where the true integral of the input function is known. In the following, the normalized integral of the input function and the kinetic parameters in the bottom row of Table I will serve as the ground truth of the simulation.

In order to examine the impact of noise on the proposed activity-subspace approach, we consider a realistic noise model. As suggested in [18], the measurement error variance is proportional to the imaged radioactivity concentration and is inversely proportional to the scan duration. Therefore, we consider the noise model that noise terms are independent Gaussian with variances

$$
\sigma^{2}\left(i, t_{j}\right)=\alpha \frac{e^{\lambda t_{j}} c_{i}\left(t_{j}\right)}{t_{j}-t_{j-1}}
$$

for $j=1, \ldots, n$, where $\alpha$ is a constant determining the noise level; $c_{i}\left(t_{j}\right)$ is the simulated noise-free TAC of voxel $i$ at time $t_{j}$; and $\lambda$ is the radioisotope decay constant. Here, we define the percent noise level as $\sqrt{\sum_{j=1}^{n} \sigma^{2}\left(i, t_{j}\right) / \sum_{j=1}^{n} c_{i}^{\prime}\left(t_{j}\right)^{2}}$, with $\left\{c_{i}^{\prime}\left(t_{j}\right)\right\}$ being the noise-free TAC for voxel $i$. The noise levels ranging from $0 \%$ to $5 \%$ are tested in the simulation.

At each noise level, we simulate 1000 runs, generating 1000 pairs of noisy TACs of the two simulated voxels. In each run, the activity-subspace approach in Section III-A is used to estimate the integral of the input function from the noisy TACs. With the estimated integral, the DVs of the two simulated voxels are estimated by LS regression of (6). The ratio between the estimated DVs is used to show the estimation performance. At each noise level, we calculate the average and standard deviation of the ratio from the 1000 simulation runs.

For the purpose of comparison, we implemented the IQML, because IQML yields the best performance among the three blind estimation algorithms compared in [13]. There are both two-compartment model and three-compartment model versions of IQML [20]. Since the simulation is based on the threecompartment model, due to the reason of no model mismatch, the three-compartment model IQML yields more accurate result than that of the two-compartment model one under noise-free case. However, the estimated DV in three-compartment IQML is very sensitive to noise even when very small noise is added and can be less accurate than that of the two-compartment IQML. We think the intuitive reason is as follows. The three-compartment model IQML does not directly estimate the DV, and it estimates a set of four parameters instead; the DV is calculated via a complicated function of the estimated parameters, and thus, its estimation error can be severely amplified. Moreover, we believe that the comparison with the two-compartment model IQML is more fair, because it has essentially the same complexity as the Logan plot in terms of the number of model parameters.
TABLE II

MeAn AND Standard DeViation of the Ratio BetweEn the Estimated DVS OF THE TWO SIMULATED VOXELS

\begin{tabular}{|c|cc|cc|}
\hline$V_{1 T} / V_{2 T}$ & \multicolumn{2}{|c|}{ Activity-subspace } & \multicolumn{2}{c|}{ IQML } \\
\hline noise level & mean & std & mean & std \\
\hline 0 & 0.3236 & 0 & 0.2801 & 0 \\
\hline 1 & 0.3247 & 0.0303 & 0.2799 & 0.0092 \\
\hline 2 & 0.3334 & 0.0592 & 0.2780 & 0.0171 \\
\hline 3 & 0.3502 & 0.0665 & 0.2759 & 0.0278 \\
\hline 4 & 0.3675 & 0.0787 & 0.2729 & 0.0387 \\
\hline 5 & 0.3921 & 0.0782 & 0.2741 & 0.0505 \\
\hline \multicolumn{5}{|c|}{ True ratio $V_{1 T} / V_{2 T}=0.3223$} \\
\hline
\end{tabular}

Therefore, we choose to compare the proposed method with the two-compartment model version of IQML. To ensure that IQML achieves its best performance, we use the true kinetic parameters as its initial. The results are shown in Table II.

In Table II, at the $0 \%$ noise level, although the integral of the input function is estimated based on the noise free TACs, the estimation is not perfect. This is because of two reasons. First, the activity-subspace approach is based on the Logan plot, which is an asymptotically linear relationship for the later part of the TACs. Since the noise-free data are simulated based on the three-compartment model, there is model mismatch between the true three-compartment model and the Logan plot. Second, the activity subspace of a voxel is spanned by the voxel TAC and its integral. With the 18 available time points of the voxel TAC, the numerical integration of the voxel TAC introduces error. For the same reason, IQML method also gives nonzero error at $0 \%$ noise level. Since the IQML method assumes two-compartment model for the entire TAC, the model mismatch is larger, and thus, a larger error is observed. At $0 \%$ noise level, since no noise is added, the estimation results from the 1000 runs are identical, which results in 0 standard deviation of the estimation error.

From Table II, we can see that the proposed activity subspace is more sensitive to noise. This result is also confirmed in [15]. In this table, we can see that the IQML method, although more robust to noise, consistently generates biased estimates. This is again because of the model mismatch between the two-compartment model assumed by IQML and the threecompartment model based on which the data are simulated. On the other hand, the propose activity-subspace approach estimates the integral of the input function, which leads to more accurate estimates of the relative DV. The only concern, so far, is the noise sensitivity.

Therefore, in order to obtain reliable estimates using the activity-subspace approach, the noise level needs to be kept small. This is our motivation for employing mPCA clustering. We also apply iterative LS method to further improve the accuracy of the estimated integral of the input function and 
simultaneously estimate the DV parameters. In the following section, we analyze the dataset from a brain PET study, and demonstrate that the activity-subspace approach, together with the mPCA and iterative LS methods, is able to estimate both the integral of the input function and the DV parametric image.

\section{REAL DATASETS}

We now examine a brain PET study. The PET data of healthy control subjects are obtained after intravenous injection of C11-labeled DASB, a radioligand used for imaging the SERT. The experimental details are the same as in [2]. In total, ten subjects are tested. A dynamic PET study is performed with a GE advance PET camera with an axial resolution [(full width at half maximum (FWHM)] of $5.8 \mathrm{~mm}$ and an in-plane resolution of $5.4 \mathrm{~mm}$. This scanner acquires 35 simultaneous slices of $4.25 \mathrm{~mm}$ thickness. A transmission scan is first obtained with twin $10 \mathrm{mCi}$ germanium-68 pin sources for 10 minutes for the purpose of attenuation correction of the emission scans. Eighteen serial dynamic PET images are acquired during the first $95 \mathrm{~min}$ after injection using the following image sequence: four $15 \mathrm{~s}$ frames, three $1 \mathrm{~min}$ frames, three $2 \mathrm{~min}$ frames, three $5 \mathrm{~min}$ frames, three $10 \mathrm{~min}$ frames, and two $20 \mathrm{~min}$ frames. All PET scans are reconstructed using the ramp-filtered back-projection technique in a $128 \times 128$ matrix, with a transaxial voxel size of $2 \times 2 \mathrm{~mm}$. All PET data are corrected for attenuation, injected dose, and radionuclide decay.

For the invasive measurement of the input function, a radial artery line is placed by an anesthesiologist. During the PET study, arterial blood samples are withdrawn every $5-7$ s during the first $2 \mathrm{~min}$, then with increasing time intervals until the end of study 95 min postinjection. Exact times of blood sampling are registered. The blood samples are centrifuged, and plasma activities are counted in a gamma counter cross-calibrated with the PET scanner every day. The exact time difference between the start of camera and the start of gamma counter is registered for decay correction. The input function is corrected for metabolized radioligand activity. For this purpose, $2 \mathrm{ml}$ arterial plasma samples are obtained 5, 15, 30,60, and $90 \mathrm{~min}$ postinjection. The extent of metabolism of C-11 DASB is determined using high performance liquid chromatography (HPLC). Missing data points of the correction function that describes the percent unmetabolized tracer are obtained by biexponential interpolation [2].

For the dynamic brain PET image data, we first perform preprocessing to identify voxels that belong to the brain region. A simple masking method is applied to sketch out the brain region. Based on the 18 dynamic PET images of the 18 time points, the sum of intensities of the observed TACs for all voxels are calculated. Voxels with intensities less than $5 \%$ of the highest intensity voxel are discarded. The remaining voxels are considered as the brain region for further estimation of the integral of the input function and the DV parametric image.

After preprocessing, the brain region is identified. In Section IV, it is shown that the proposed activity-subspace approach is sensitive to noise. Therefore, we cannot directly apply the activity-subspace approach based on the noisy voxel TACs. To reduce the noise, we take voxels from the brain regions of all slices, and apply the mPCA approach in Section III-B to group the brain voxel TACs into two clusters. The activity of each cluster is represented by the average of voxel TACs within this cluster. Through averaging, the noise is reduced. Then, the integral of the input function is estimated using the activity-subspace approach, which operates on the cluster average TACs. In Fig. 3(a), we use the data for subject 2 as an example. The estimated integral of the input function is shown by a solid line labeled with stars. The normalized integral of the measured input function is shown by the solid line labeled with circles. To improve the accuracy of the estimated integral of the input function, the iterative LS method in Section III-C is applied to iteratively refine the estimated integral of the input function based on the TACs of all brain voxels. In Fig. 3(a), the refined estimate of the integral is shown by the solid line labeled with triangles.

After obtaining the estimated integral of the input function, we further estimate the relative DVs. Based on the estimated integral of the input function, the relative DV parameters are obtained by linear regression of (7) for each voxel [18]. For comparison, the relative DV parameters are also calculated based on the normalized integral of the measured input function. In Fig. 3(b), the scatter plot of the relative DV parameters is shown, where each dot corresponds to one voxel, the horizontal and vertical axes represent the estimated parameters with the measured and estimated integral of the input function. The scatter plot closely follows the 45 degree line, which indicates high estimation accuracy. To quantify the estimation accuracy, we compute the normalized relative distance (PM) between the true and estimated parametric images. PM is defined to compare the two parametric images

$$
\mathrm{PM}=\frac{1}{N} \sum_{i=1}^{N}\left|\frac{\left(\hat{V}_{i T}-\bar{V}_{i T}\right)}{\bar{V}_{i T}}\right|
$$

where $\bar{V}_{i T}$ is the relative $\mathrm{DV}$ obtained by the normalized integral of the measured input function, $\hat{V}_{i T}$ is the estimated relative DV based on the estimated integral of the input function, and $N$ is the total number of voxels in the brain region. For subject 2 , $\mathrm{PM}=0.012$, meaning that the DV image based on the estimated integral contain $1.2 \%$ error, compared with the DV image based on the measured input function. In Fig. 3(c) and (d), we use slice 15 as example to show the DV parametric images based on the measure and estimated integral of the input function. The difference image is shown in Fig. 3(e). The images shown are after three-by-three neighborhood median filtering. In Fig. 3(c) and (d), both images show the expected high binding in the region of basal ganglia and the midbrain.

In total, we analyze ten subjects. In Table III, we show the PM that quantifies the difference between the DV parametric images based on the estimated and measured integrals of the input function. From Table III, the PM error for most of the subjects are small, and the average PM is 5.2\%. Excluding subject 8 with extremely large PM, the average error reduces to $2.8 \%$. To better illustrate the estimation error, in Fig. 4, we 


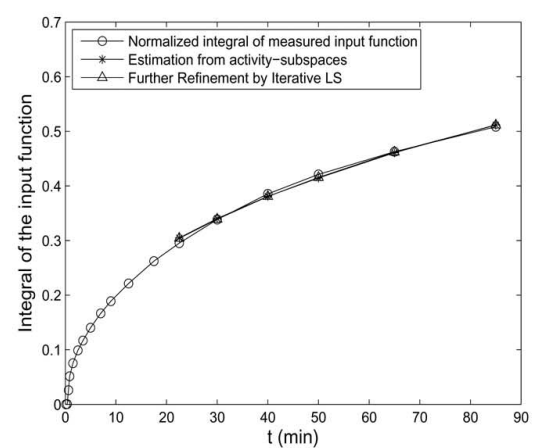

(a)

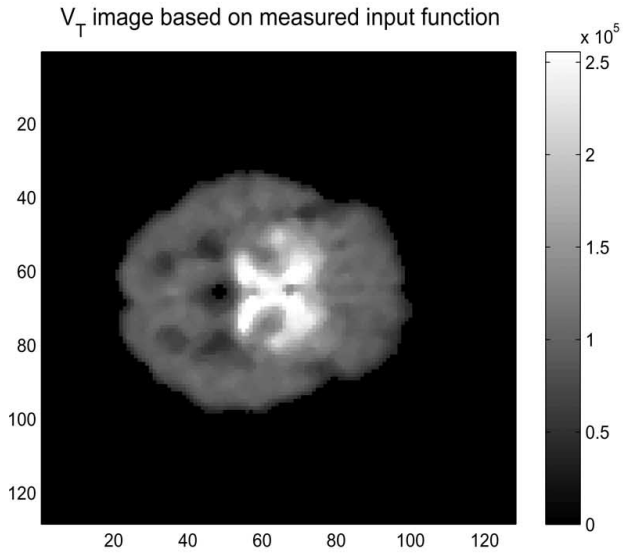

(c)

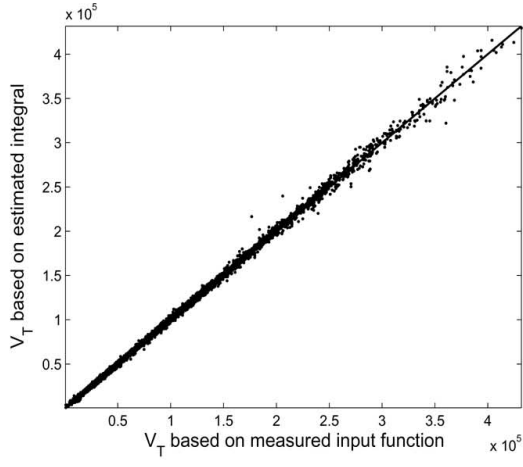

(b)

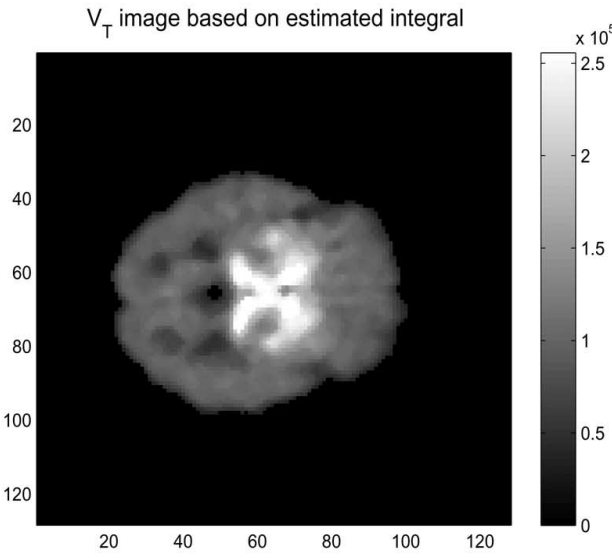

(d)

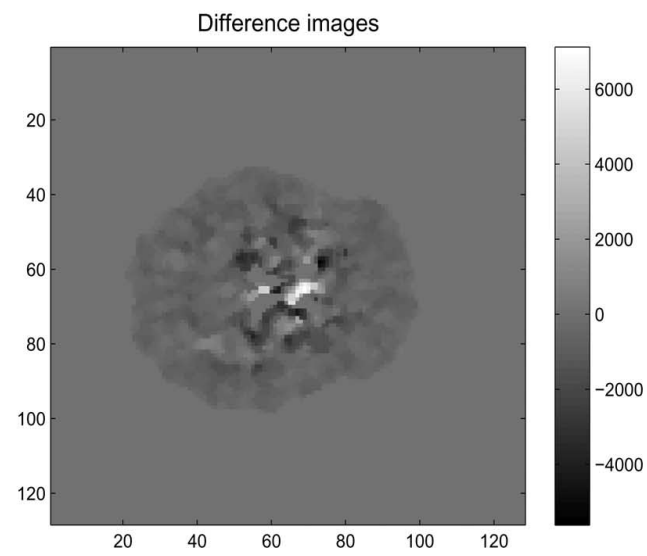

(e)

Fig. 3. Results from subject 2. (a) Normalized the integral of the measured input function, the estimated integral of the input function based on activity subspaces, and the refined results by further applying iterative LS. (b) Scatter plot of the estimated DVs based on the measured and estimated integrals. (c) DV parametric image of slice 15 based on the measured integral. (d) DV parametric image based on the estimated integral. (e) Difference between Fig. (c) and (d). The images shown are after three-by-three neighborhood median filtering.

\section{TABLE III}

PM METRIC THAT QUANTIFIES THE DIFFERENCE BETWEEN THE DV PARAMETRIC IMAge BASED ON THE PROPOSED METHOD AND THAT BASED ON THE MEASURED INPUT FUNCTION

\begin{tabular}{|c|c||c|c|}
\hline subject & $P M$ & subject & $P M$ \\
\hline 1 & 0.024 & 6 & 0.018 \\
\hline 2 & 0.012 & 7 & 0.031 \\
\hline 3 & 0.038 & 8 & 0.261 \\
\hline 4 & 0.006 & 9 & 0.041 \\
\hline 5 & 0.068 & 10 & 0.018 \\
\hline
\end{tabular}

show the estimated integral and the scatter plot of estimated DVs: (a) and (b) for subject 1, whose PM is $2.4 \%$; (c) and (d) for subject 9, whose PM is $4.1 \%$; (e) and (f) for subject 8 , whose PM is $26.1 \%$. For subject 8 , the proposed method gives an incorrect estimate of the integral. The scatter plot in Fig. 4(f) shows a linear relationship. The estimated DV image is of lower contract, compared with the case where the input function is measured. Therefore, the estimated DV image is qualitatively meaningful but quantitatively incorrect. For the 


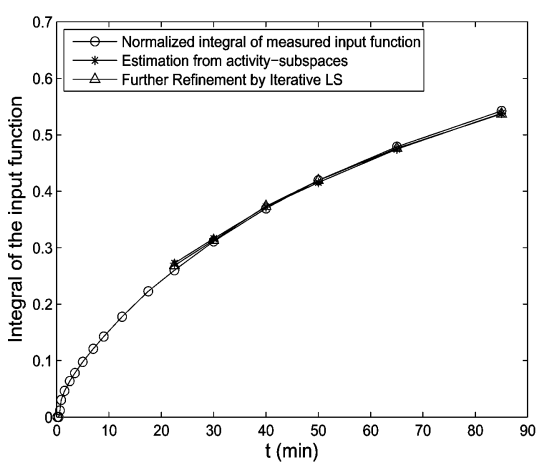

(a)

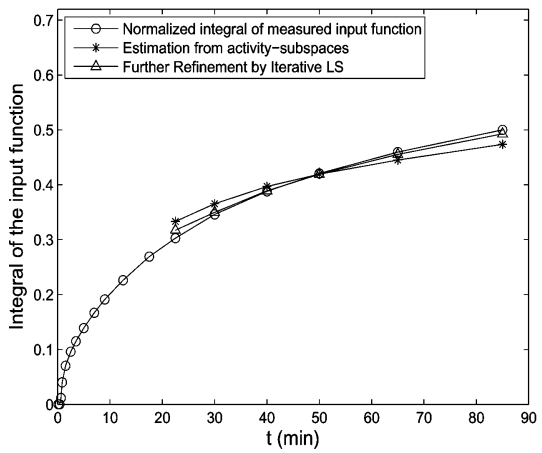

(c)

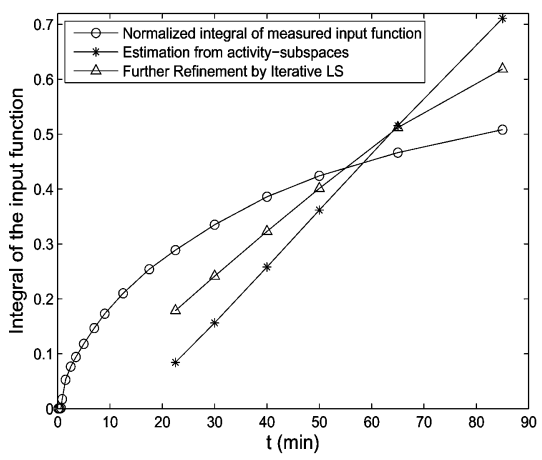

(e)

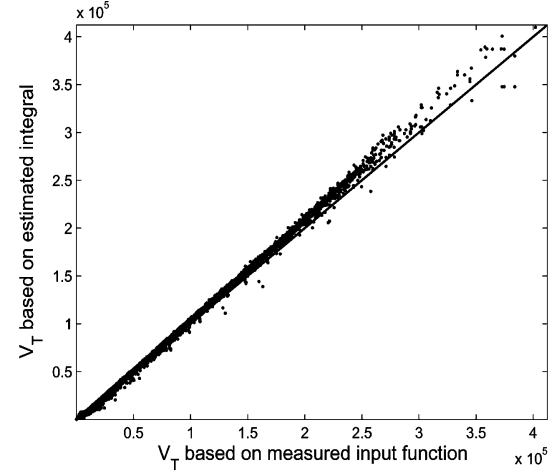

(b)

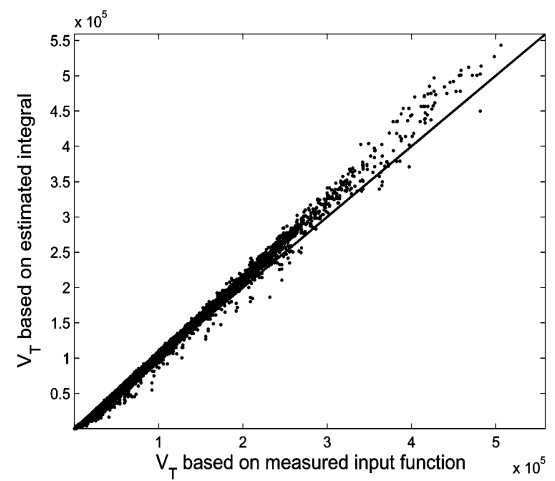

(d)

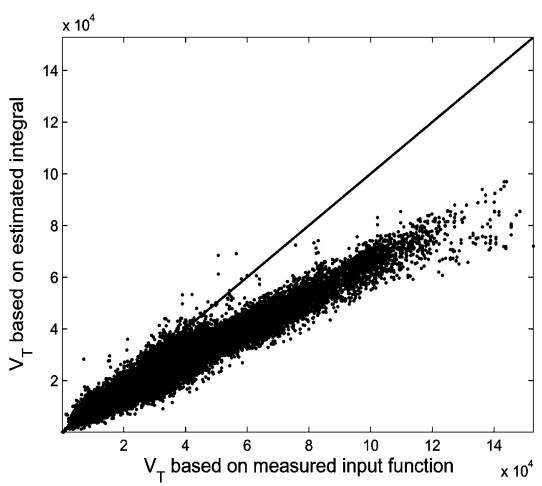

(f)

Fig. 4. Left column is the measured and estimated integrals of the input function. The right column is the scatter plots of the estimated DV parameters. (a) and (b) Correspond to subject 1. (c) and (d) Correspond to 9. (e) and (f) Correspond to subject 8.

other nine subjects, the proposed method is able to estimate the integral of the input function and the DV parametric image, giving an average error of $2.8 . \%$.

\section{DISCUSSION}

In this paper, we presented an activity-subspace approach for estimating the integrated input function and the relative DV parametric image. Rather than providing absolute quantification, the proposed method only estimates the relative DV within a scale factor. The scale factor is a common issue to any blind identification methods where no assumptions are made on the unknown input function. When analyzing PET data of a single subject, the relative DV is useful for comparisons across different slices or ROIs. For comparisons across subjects, an important issue, absolute quantification of the DV, needs to be addressed, and therefore, additional assumptions or additional data are required. For example, if the total amount of dosage is controlled such that the integrals of the input functions of different subjects are the same, the proposed method can be applied. If the artery is included in the dynamic images, after metabolite correction, the ratio between the input functions of two subjects can be inferred from the intensities of the artery voxels, and we can further use the ratio to adjust the estimated relative DVs of the two subjects. Another possible option for achieving absolute quantification is to take a single arterial sample during scanning (or a venous sample if the relationship between arterial concentration and venous concentration is well established) to provide 
a reasonable estimate of the scale factor, though more research efforts may be needed for determining the optimal time point to take the sample.

In the analysis of the PET DASB study in Section V, the measured input function is considered as the gold standard. Frankle et al. [4] shed light on the validity of this gold standard. It was shown in [4] that the estimated kinetic parameters by several methods were consistent, including the Logan plot [17] and a reference tissue method, simplified reference tissue method (SRTM) [9]. Logan plot assumed knowing the measured input function, while SRTM assumed a reference region. Although the two methods started from different assumptions, the estimated kinetic parameters were consistent, indicating that both the measured input function and the reference region were correct. In Section V, we observe that, in most subjects, the estimated integral of the input function is close to the normalized integral of the measured input function. This observation enhanced our belief that it is reasonable to regard the measured input as the gold standard in this study.

A challenging question general to blind identification methods is that how to tell whether the estimates are correct or not, when the true parameters are unknown. We are lack of a theoretical answer to this question. Heuristically, it is observed from Fig. 4(e) that the estimated integral of the input function is incorrect since the shape of the integral is clearly incorrect. For more challenging cases where the shape of the estimated integral may look correct, though not observed in our study, one possible solution is to examine the statistical stability via bootstrap, similar to the reproducibility idea in [4]. We can generate bootstrapped datasets and estimate the integral from each bootstrapped dataset. If the estimated integrals from different bootstrapped data are statistically consistent, we can have certain confidence in the result; if otherwise, this can be an indication of failure.

\section{CONCLUSION}

Of interest in this paper is the estimation of the total DV parametric image in PET study when the knowledge of the plasma input function is not available. In this paper, we have presented a novel concept of activity subspace, and derived the method for estimating the integral of the input function by exploring the intersections of the activity subspaces spanned by the voxel cluster TACs and their integrals. No prior information regarding the input function or the reference region is needed in the proposed method. We presented the mPCA to group the brain voxels into clusters so that the noise is reduced and the activitysubspace approach is able to estimate the integral of the input function more reliably. An iterative LS method is incorporated to further improve the accuracy of the estimated integral of the input function. Results from a PET brain study show that, for the noninvasive studies when the measurement of plasma input function or a reference region is unavailable, the proposed activity-subspace approach, together with the mPCA method and the iterative LS method, is able to efficiently estimate the integral of the input function and the DV parametric images. In a real dynamic PET dataset of ten subjects, the proposed method achieved an average error of $5.2 \%$ compared with the case where the true input function is measured and known.

\section{APPENDIX}

An EM algorithm for estimating mPCA model is proposed in [19]. The algorithm can be summarized as follows. Given the model parameters from initialization or previous iteration, the probability of observation $y_{i}$ conditioning on the $k$ th probabilistic PCA model parameters can be calculated

$$
\begin{aligned}
p\left(y_{i} \mid k\right) & =\int p\left(y_{i} \mid x, k\right) p(x \mid k) d x \\
& =(2 \pi)^{-d / 2}\left|C_{k}\right|^{-1 / 2} e^{-\frac{1}{2}\left(y_{i}-\mu_{k}\right)^{T} C_{k}^{-1}\left(y_{i}-\mu_{k}\right)}
\end{aligned}
$$

where $C_{k}=\sigma_{k}^{2} I+W_{k} W_{k}^{T}$. Given the prior probabilities $p(k)$ of a set of $K$ probabilistic PCA models, the marginal probability of observation $y_{i}$ is

$$
p\left(y_{i}\right)=\sum_{k=1}^{K} p\left(y_{i} \mid k\right) p(k)
$$

and the posterior probability of the $k$ th probabilistic PCA model can be expressed as

$$
p\left(k \mid y_{i}\right)=\frac{p\left(y_{i} \mid i\right) p(k)}{p\left(y_{i}\right)} .
$$

Therefore, the posterior probability can be used for the classification purpose.

In the M-step, the update of model parameters can be summarized as follows. Suppose that there are in total $N$ voxels under consideration.

$$
\begin{aligned}
\widetilde{p}(k) & =\frac{1}{N} \sum_{i=1}^{N} p\left(k \mid y_{i}\right) \\
\widetilde{\mu}_{k} & =\frac{\sum_{i=1}^{N} p\left(k \mid y_{i}\right) y_{i}}{\sum_{i=1}^{N} p\left(k \mid y_{i}\right)} \\
\widetilde{W}_{k} & =S_{k} W_{k}\left(\sigma_{k}^{2} I+M_{k}^{-1} W_{k}^{T} S_{k} W_{k}\right)^{-1} \\
\widetilde{\sigma}_{k}^{2} & =\frac{1}{d} \operatorname{tr}\left(S_{k}-S_{k} W_{k} M_{k}^{-1} \widetilde{W}_{k}^{T}\right)
\end{aligned}
$$

where $\widetilde{p}(k), \widetilde{\mu}_{k}, \widetilde{W}_{k}$, and $\widetilde{\sigma}_{k}^{2}$ are the updated model parameters for the $k$ th probabilistic PCA model in the mixture, and

$$
\begin{aligned}
S_{k} & =\frac{1}{\widetilde{p}(k) N} \sum_{i=1}^{N} p\left(k \mid y_{i}\right)\left(y_{i}-\widetilde{\mu}_{k}\right)\left(y_{i}-\widetilde{\mu}_{k}\right)^{T} \\
M_{k} & =\sigma_{k}^{2} I+W_{k}^{T} W_{k}
\end{aligned}
$$

\section{REFERENCES}

[1] S. R. Cherry, "Fundamentals of positron emission tomography and applications in preclinical drug development," J. Clin. Pharmacol., vol. 41, pp. 482-491, 2001.

[2] L. Kerenyi, G. A. Ricaurte, D. J. Schretlen, U. McCann, J. Varga, W. B. Mathews, H. T. Ravert, R. F. Dannals, J. Hilton, D. F. Wong, and Z. Szabo, "Positron emission tomography of striatal serotonin transporters in Parkinson's disease," Arch. Neurol., vol. 60, pp. 1223-1229, 2003. 
[3] R. N. Gunn, S. R. Gunn, and V. J. Cunningham, "Positron emission tomography compartmental models," J. Cereb. Blood Flow Metab., vol. 21, no. 6, pp. 635-652, 2001.

[4] W. G. Frankle, M. Slifstein, R. N. Gunn, Y. Huang, D. R. Hwang, E. A. Darr, R. Narendran, A. Abi-Dargham, and M. Laruelle, "Estimation of serotonin transporter parameters with 11C-DASB in healthy humans: Reproducibility and comparison of methods," J. Nucl. Med., vol. 47, pp. 815-826, 2006.

[5] V. J. Cunningham and A. A. Lammertsma, "Radioligand studies in brain: Kinetic analysis of PET data," Med. Chem. Res., vol. 5, pp. 79-96, 1994.

[6] J. H. Meyer and M. Ichise, "Modeling of receptor ligand data in PET and SPECT imaging: A review of major approaches," J. Neuroimaging, vol. 11, no. 1, pp. 30-39, 2001.

[7] J. Correia, "Editorial: A bloody future for clinical PET," J. Nucl. Med., vol. 33, pp. 620-622, 1992.

[8] K. Chen, D. Bandy, E. Reiman, S. C. Huang, M. Lawson, D. Feng, L. S. Yun, and A. Palant, "Noninvasive quantification of the cerebral metabolic rate for glucose using positron emission tomography, ${ }^{1} 8 \mathrm{~F}$ fluoro-2-deoxyglucose, the patlak method, and an image-derived input function," J. Cereb. Blood Flow Metab., vol. 18, pp. 716-723, 1998.

[9] A. A. Lammertsma and S. P. Hume, "Simplified reference tissue model for PET receptor studies," Neuroimage, vol. 4, no. 3, pp. 153-158, 1996.

[10] R. N. Gunn, A. A. Lammertsma, S. P. Hume, and V. J. Cunningham, "Parametric imaging of ligand-receptor binding in PET using a simplified reference region model," Neuroimage, vol. 6, no. 4, pp. 279-287, 1997.

[11] K. P. Wong, D. Feng, S. R. Meikle, and M. J. Fulham, "Simultaneous estimation of physiological parameters and input function-In vivo PET data," IEEE Trans. Inf. Technol. Biomed., vol. 5, no. 1, pp. 67-76, Mar. 2001.

[12] Z. J. Wang, Z. Szabo, P. Lei, J. Varga, and K. J. R. Liu, "A Factor-image framework to quantification of brain receptor dynamic PET studies," IEEE Trans. Signal Process., vol. 53, no. 9, pp. 3473-3487, Sep. 2005.

[13] D. Y. Riabkov and E. Di Bella, "Estimation of kinetic parameters without input functions: Analysis of three methods for multichannel blind identification," IEEE Trans. Biomed. Eng., vol. 49, no. 11, pp. 1318-1327, Nov. 2002.

[14] Z. J. Wang, P. Qiu, K. J. R. Liu, and Z. Szabo, "Model-based receptor quantization analysis for PET parametric imaging," in Proc. IEEE Eng. Med. Biol. Conf., Sep. 2005, pp. 5908-5911.

[15] M. Naganawa, Y. Kimura, J. Yano, M. Mishina, M. Yanagisawa, K. Ishii, K. Oda, and K. Ishiwata, "Robust estimation of the arterial input function for logan plots using an intersectional searching algorithm and clustering in positron emission tomography for neuroreceptor imaging," NeuroImage, vol. 40, no. 1, pp. 26-34, 2008.

[16] P. Qiu, Z. J. Wang, and K. J. R. Liu, "Mixture principal component analysis for distribution volumn parametric imaging in brain PET studies," in Proc. IEEE Int. Symp. Biomed. Imag. (ISBI), Apr. 2006, pp. 928-931.

[17] J. Logan, J. S. Fowler, N. D. Volkow, A. P. Wolf, S. L. Dewey, D. J. Schlyer, R. R. MacGregor, R. Hitzeman, B. Bendiriem, S. J. Gatley, and D. Cristman, "Graphical analysis of reversible radioligand binding from time-activity measurements applied to $\left[N-{ }^{11} C-\right.$ methyl $]-(-)-$ cocaine PET studies in human subjects," J. Cereb. Blood Flow Metab., vol. 10, pp. 740-747, 1990.

[18] M. Ichise, H. Toyama, R. B. Innis, and R. E. Carson, "Strategies to improve neuroreceptor parameter estimation by linear regression analysis," $J$. Cereb. Blood Flow Metab., vol. 22, pp. 1271-1281, 2002.

[19] M. E. Tipping and C. M. Bishop, "Mixtures of probabilistic principal component analyzers," Neural Comput., vol. 11, no. 2, pp. 443-482, 1999.

[20] D. Y. Riabkov and E. Di Bella, "Blind identification of the kinetic parameters in three-compartment models," Phys. Med. Biol., vol. 49, pp. 639664, 2004.

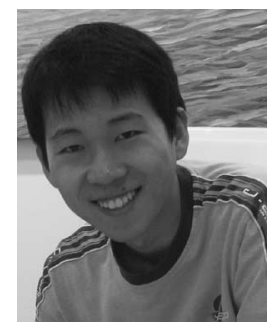

Peng Qiu (M'08) received the B.S. degree from the University of Science and Technology of China, Hefei, China, in 2003, and the Ph.D. degree from the University of Maryland, College Park, in 2007, both in electrical engineering.

$\mathrm{He}$ is currently a Postdoctoral Fellow of the Integrative Cancer Biology Program, School of Medicine, Stanford University, Stanford, CA. His current research interests are in bioinformatics and computational biology, focusing on signal processing and machine learning.

Dr. Qiu received the Electrical and Computer Engineering (ECE) Distinguished Dissertation Fellowship from the University of Maryland.

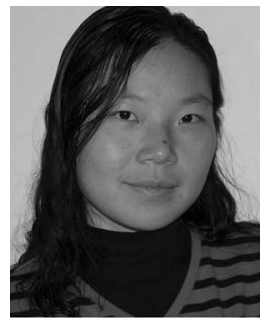

Z. Jane Wang (M'02) received the B.Sc. degree (with the highest honor) from Tsinghua University, Beijing, China, in 1996, and the M.Sc. and Ph.D. degrees from the University of Connecticut, Storrs, in 2000 and 2002 , respectively, all in electrical engineering.

She had been a Research Associate in the Electrical and Computer Engineering Department, University of Maryland, College Park. Since August 2004, she has been an Assistant Professor in the Department of Electrical and Computer Engineering, University of British Columbia (UBC), Vancouver, BC, Canada. Her current research interests include the broad areas of statistical signal processing, with applications to multimedia security and biomedical signal modeling and imaging. She is an Associate Editor of the European Association for Signal Processing (EURASIP) Journal on Bioinformatics and Systems Biology.

Dr. Wang coreceived the EURASIP Best Paper Award in 2004 and the IEEE Signal Processing Society Best Paper Award in 2005. She is an Associate Editor of the IEEE TRANSACTIONS ON MULTIMEDIA. She is a member of the IEEE Signal Processing Society (SPS) Bio-Imaging Signal Processing Technical Committee.

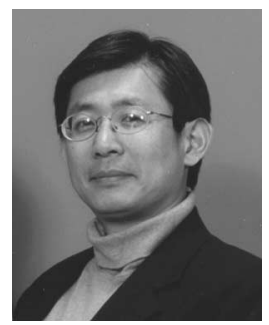

K. J. Ray Liu (F'03) received the B.S. degree from the National Taiwan University, Taiwan in 1983, and the $\mathrm{Ph} . \mathrm{D}$. degree from the University of California, Los Angeles in 1990, both in electrical engineering.

$\mathrm{He}$ is a Distinguished Scholar-Teacher of the University of Maryland, College Park. He is also the Associate Chair of Graduate Studies and Research of the Electrical and Computer Engineering Department and leads the Maryland Signals and Information Group conducting research encompassing broad aspects of information technology including communications and networking, information forensics and security, multimedia signal processing, and biomedical technology. He is the founding Editor-in-Chief of the European Association for Signal Processing (EURASIP) Journal on Applied Signal Processing. His recent books include Cooperative Communications and Networking (Cambridge University Press, 2008); Resource Allocation for Wireless Networks: Basics, Techniques, and Applications (Cambridge University Press, 2008); Ultra-Wideband Communication Systems: The Multiband OFDM Approach (IEEE-Wiley, 2007); Network-Aware Security for Group Communications (Springer-Verlag, 2007); Multimedia Fingerprinting Forensics for Traitor Tracing (Hindawi, 2005); and Handbook on Array Processing and Sensor Networks (IEEE-Wiley), 2009.

Dr. Liu is the recipient of numerous honors and awards, including Best Paper Awards from the IEEE Signal Processing Society (twice), the IEEE Vehicular Technology Society, and the EURASIP, the IEEE Signal Processing Society Distinguished Lecturer, the EURASIP Meritorious Service Award, and the National Science Foundation Young Investigator Award. He has also received various teaching and research recognitions from the University of Maryland, including university-level Invention of the Year Award, and the Poole and Kent Senior Faculty Teaching Award and the Outstanding Faculty Research Award, both from the A. James Clark School of Engineering Faculty. He is the Vice President - Publications and a member of the Board of Governor of the IEEE Signal Processing Society. He was the Editor-in-Chief of the IEEE SIGNAL PRocessing Magazine.

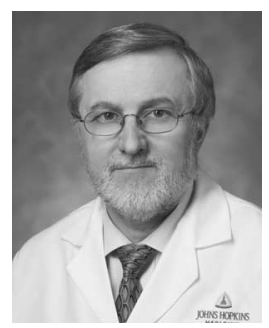

Zsolt Szabo received the M.D. degree from the Medical School, University of Belgrade, Belgrade, Serbia, and the Ph.D. degree from the University of Düsseldorf/Nuclear Research Center Julich, Düsseldorf, Germany.

He pursued additional graduate training at the University of Dusseldorf Germany and the Johns Hopkins University in the USA. He is currently a Faculty Member and an Attending Nuclear Medicine Physician at the Johns Hopkins Medical Institutions, Baltimore, MD. He has authored or coauthored 74 articles published in peer-reviewed journals and 40 chapters in medical textbooks. He is a coeditor of the textbook Principles of Nuclear Medicine. He has also been a reviewer for study sections and scientific journals. His current research interests include investigation of central serotonin, dopamine transporters, peripheral angiotensin, and endothelin receptors with positron emission tomography (PET).

Dr. Szabo was a recipient of multiple previous and present National Institutes of Health $(\mathrm{NIH})$ grants. He was certified in nuclear medicine by the German Medical Association and by the US Board of Nuclear Medicine. 